\title{
Measuring Complexity of SAP Systems
}

\author{
Ilja Holub and Tomas Bruckner \\ University of Economics, náměstí Winstona Churchilla 1938/4, 13067 \\ Praha 3, Czech Republic \\ \{ilja.holub, tomas.bruckner\}@vse.cz
}

\begin{abstract}
The paper discusses the reasons of complexity rise in ERP system SAP R/3. It proposes a method for measuring complexity of SAP. Based on this method, the computer program in ABAP for measuring complexity of particular SAP implementation is proposed as a tool for keeping ERP complexity under control. The main principle of the measurement method is counting the number of items or relations in the system. The proposed computer program is based on counting of records in organization tables in SAP.
\end{abstract}

Keywords: Complexity, ERP, SAP, measurement, information system, business.

\section{Introduction}

In this paper we discuss the approaches to complexity and show that several methodologies used for Information and communication technology (ICT) and information systems (IS) management do not handle the topic of complexity well. Then we discuss the complexity of SAP ERP ${ }^{1}$ system $^{-1}$ and the reasons for rising complexity in SAP implementations. Afterwards we propose a method and a program for measuring complexity of ERP systems and describe a verification of the method on a certain business case.

There are several decisions during the lifecycle of an SAP System that influence the complexity of the systems. These decisions are made by project manager, by IT managers or by team members. The goal of our paper is to give a tool for the decision makers, which can measure the complexity at a certain point. It could be used by project manager or solution architect during the implementation to control the complexity and prevent the growth of it.

\section{Related Work on Measuring Complexity}

A good theoretical basis for describing and exploring complexity can be found in the mathematical graph theory [1], [2], which can be applied to some IS/ICT models to facilitate their quantification and subsequent comparison and, in some cases, even their simplification. Publications that come closest to the topic of business information system complexity deal with using metrics to measure complexity, such as information flow metrics [3], Halstead's method or functional point analysis [4], or a simple number of source code lines [5]. These methods are focused on software development and are less suitable to handle SAP systems, where more settings are done by customization and system configuration. There are also publications dealing with the impacts of complexity on system security and maintainability. Their objective is to

\footnotetext{
${ }^{1}$ SAP ERP - Enterprise Resource Planning software of SAP company (www.sap.com)
} 
describe certain aspects of complexity [6] and/or to point out the negative impacts of complexity on the business [5].

The topic of managing information system complexity is discussed, for example, by John Maeda in his book "The Law of Simplicity", where he defines ten rules of simplicity [7]. ERP systems are specifically discussed in "A Metric for ERP Complexity" [3], which counts process inputs and outputs and works with the concepts of internal and external module complexity, applying data flow complexity according to [8]. Best practices for simplicity of IS are described in the book "Managing Complexity of Information Systems" [9].

In several methodologies used to manage ICT and information system projects the issue of complexity is rather insufficiently discussed. Although some of them do mention the risks and costs arising from high complexity, none of them presents a method how to handle or even reduce complexity. We prepared a summary overview of the most frequently used methodologies (see Table 1).

Table 1. Occurrences of the word "complex" in various methodologies; source: authors

\begin{tabular}{|l|c|c|c|c|}
\hline \multicolumn{1}{|c|}{ Methodology Title } & Pages & $\begin{array}{c}\text { Occurrence of the } \\
\text { word "complex" }\end{array}$ & $\begin{array}{c}\text { Occurrence of the } \\
\text { word "simplify" }\end{array}$ & $\begin{array}{c}\text { Number of solutions } \\
\text { described }\end{array}$ \\
\hline The Val IT Framework 2.0 & 116 & 6 & 1 & 0 \\
\hline TOGAF Version 9 & 778 & 74 & 11 & 5 \\
\hline Rational Unified Process & 21 & 3 & 0 & 0 \\
\hline Prince 2 & 326 & 26 & 0 & 0 \\
\hline PMBOK & 403 & 35 & 1 & 0 \\
\hline COBIT5 & 94 & 9 & 0 & 0 \\
\hline
\end{tabular}

From among the methodologies listed here, TOGAF is the one that mentions complexity most often, and it is the only methodology that discusses this topic at least briefly. The agile methodologies are not widely spread within the SAP implementation projects, therefore we skip these.

The proposed SAP measurement method is a part of a general complexity management methodology [10] prepared by authors, which details are beyond the scope of this paper.

The important principle is to keep the managing complexity itself simple. The managing complexity including measurement should not bring another complexity, so it should be kept simple and simplified even at the expense of accuracy or precision. The reason for measuring complexity is to have a metric, which can measure not the actual absolute complexity of the system, but relative complexity in time or before and after a change, and which is therefore useful for managing complexity and keeping the system as simple as possible.

The nature of complexity is multidimensional, because it impacts different stakeholders: users, process and/or company owners, developers, or system administrators. We call this different views, content dimensions, and do measure the complexity for every dimensions separately. [10] In every project and for every information system the importance of content dimensions is very different.

When measuring system complexity, the first step is to select relevant content dimensions D and the IS lifecycle phase $f$, using which we wish to measure complexity. Let us designate their number as $d$. For dimension $i$ in phase $f$ we can define complexity $\mathrm{C}_{\mathrm{f}}\left(\mathrm{D}_{\mathrm{i}}\right)$ as the number of entities (elements and/or links) of its model.

Let us define system complexity $\mathrm{C}(\mathrm{S})$ as a sum of the complexities of each of its dimensions $\mathrm{C}\left(\mathrm{D}_{\mathrm{i}}\right)$. Thus, complexity $\mathrm{C}$ of system $\mathrm{S}$ in phase $f$ is the number of entities (elements and/or links) in the models of each of its content dimensions (D) selected in the given phase; $d$ shall be the total number of dimensions considered.

$$
C_{f}(S)=\sum_{i=1}^{d} C_{f}\left(D_{i}\right)
$$


The formula (1) allows us to quantify the complexity of a system or its parts, and in practice it can be used to compare various systems or solution alternatives that meet the given requirements, yet there is no objective criterion why one of them should be selected.

\section{Complexity of SAP}

In this paper we research the SAP ERP information system in terms of complexity and use it as an example of how the complexity of an information system can be defined and calculated, what its causes are, and what impact system complexity has. We discuss the context of SAP complexity and propose a simple way to measure it. Resulting measure is meant to be used to control unexpected complexity growth during several phases of information system lifecycle.

\subsection{Products and Policy of SAP}

According to Forbes Global $2000^{2}$, in 2015 the company SAP was the largest European and third largest global software manufacturer [11]. Its main product - SAP R/3, which replaced the original SAP R/2 in 1992 and since 2013 has been named SAP ERP Central Component (ECC) is today the number one ERP system and has been installed by 50000 customers in 25 different sectors, in 37 languages in 45 countries. It covers all key processes from purchasing, manufacturing, sales, and service to finances and human resources [12]. This ERP system was updated in recent years with further systems such as Customer Relationship Management (CRM), Supplier Relationship Management (SRM), Supply Chain Management (SCM), Product Lifecycle Management (PLM), SAP Human Capital Management (HCM) and other systems which specialise in the most important business processes. Today all these systems (components) along with the original ERP are called SAP Business Suite. For customers that purchased these systems, the given processes were detached from the original ERP R/3 into multiple systems. This increased the demands for integration as well as the requirements for consultants and users, who have to deal with further systems. In parallel to this, SAP is also putting further technologies on the market, such as Business Intelligence (BI) solutions and mobile and cloud services, which further complicate the whole architecture of enterprise information systems. This broad portfolio allows SAP to offer customers in various sectors specialised solutions and collect revenue from licences for multiple systems, but, on the other hand, it increases the demands on customers, who must hire specialists to implement and maintain these systems. Despite the fact that the software comes from a single supplier, it is so complicated that it can only rarely be covered by a single person. Thus, even for the IT departments of large companies this means substantial external costs; for small and medium enterprises it is often an unsolvable problem. Attention is drawn to this issue by the organisation $\mathrm{DSAG}^{\mathbf{3}}$, which brings together Germanspeaking clients of SAP.

\subsection{Zurück in die Zukunft ${ }^{4}$ [13]}

At the 13th annual conference of DSAG on 25 September 2012, its chairperson, Dr. Karl Liebstückl, criticised the number of new products by SAP and appealed for a return to a single integrated solution that could cover all business processes:

\footnotetext{
${ }^{2}$ Forbes Global 2000 is an annual ranking of the top 2000 public companies in the world according to Forbes magazine. The evaluation is based on a combination of four metrics: sales, profits, assets and market value.

${ }^{3}$ Deutschsprachige SAP-Anwendergruppe is an organisation of German-speaking SAP users that was formed in 1997 with its headquarters in Walldorf, and in 2012 it associated over 2700 SAP clients. It is the largest independent organisation of SAP users.

${ }^{4}$ Back to the future - The motto which according to Dr Liebstückl should become the starting point for cooperation between DSAG and SAP in the coming months.
} 
"SAP hatte das im Griff, als es nur ein Produkt gab" [14]

"DSAG calls for SAP's IT solutions, such as SAP Business Suite, mobile and cloud applications and others, to be integrated into a better and simpler single system with ERP at its centre. SAP should focus on its own ERP and better integrate new products. This is the only way to prevent complexity. ...

From a user perspective there is a desperate need to simplify end-to-end processes. The first step should be returning the functions of SAP Business Suite back into SAP ERP."

Another problem of complexity growth is SAP's licensing policy, which prevents effective use of technology and makes it difficult for customers to decommission or downsize older or unnecessary systems. DSAG states the following on this in its press release [14]:

"SAP firmly lays down the configuration of contracts so that companies are bound for years, regardless of how the economy and markets develop. (Once under a service contract, always under a service contract. $)^{6}$ Partial cancellation or decommissioning is thus no longer possible."

It is important to realise that information and communication technologies at an enterprise come from multiple suppliers, and though SAP is often the primary supplier, it is not the only one; and the issue of system integration of various suppliers is escalated by the necessity of integrating various SAP solutions amongst themselves.

\subsection{SAP Systems and the Growth of Their Complexity}

The complexity of the SAP system is determined in part by the selection of the set of business processes that are to be supported by the system and also by which systems or modules are to be implemented.

System lifecycle. The basis for implementing a SAP system is an analysis of the business processes and needs for supporting them on the part of the information system. The standard implementation procedure starts with an analysis of the business processes and IS requirements, based on which, according to the ASAP methodology, a target concept (Blueprint) is created, which serves as the assignment for setting up the system. This document describes the structure of the enterprise and includes the basic organisational structures of the individual SAP modules, the types of documents and the types of master data.

Standard software selection. A crucial decision influencing the complexity of the ICT at an enterprise is the choice of standard ERP system. The SAP itself offers a broad portfolio of products that use various technological platforms, are focused on various branches of industry, or cover specialised process groups within an enterprise. A description of all such products and their complexity is beyond the scope of this article - here we will focus solely on the most widespread of them, to which SAP owes its success: SAP ERP. Deciding just for SAP ERP means the resulting system will have the least possible complexity. In its basic configuration, before applying user settings, SAP ERP contains only model organisational structures and document types. Depending on the description of the business structures and processes, customisation must be carried out, along with potential additional programming of certain specific customer requirements.

Setting up (customizing) the system. A key attribute of SAP systems is the extensive possibilities for user customisation, which can adapt the system to a broad range of business processes and variants thereof, from purchasing to manufacturing to sales, including warehouse management, quality management, and last but not least controlling and accounting. System could be configured for all sizes from small or medium company to multinational concerns. This property, which is imperative for large companies, also leads to such a level of complexity that it has been shown to be a knock-out blow for small businesses that could also work with SAP, but merely setting up the system is so demanding that it is beyond their capabilities. For small and

\footnotetext{
${ }^{5}$ SAP managed at the time when there was only one product. Meant SAP R/3 - SAP ERP.

${ }^{6}$ The service contract with SAP is paid annually as a certain percentage of the original software purchase price. (Author's note)
} 
medium enterprises, complexity leads to costs and risks that could be avoided by reducing complexity.

\subsection{Measuring the Complexity of SAP Systems}

The complexity of SAP system changes over its lifecycle, and if it is not specifically managed, it grows. To calculate it we use a program written in the $\mathrm{ABAP}^{7}$ programming language.

Which parameters influence the complexity of SAP systems? The complexity of the system grows during its customisation. The SAP system contains tens of thousands of customisation tables, the entries of which define the system's settings and determine its complexity. The basic setting that determines all the other settings is the company's organisational structure setting. Like all other settings it takes place in an SPRO transaction ${ }^{8}$.

Table 2 displays the pertinent customisation tables for the individual branches of customisation tree in SAP R/3 for organisational structure.

Table 2. SAP tables defining an enterprise's organizational structure

\begin{tabular}{|c|c|c|}
\hline Module & Orgstructure & Table \\
\hline \multirow{7}{*}{$\begin{array}{l}\text { Financial } \\
\text { Accounting }\end{array}$} & Define company & T880 \\
\hline & Define Credit Control Area & T014 \\
\hline & Edit, Copy, Delete, Check Company Code & T001 \\
\hline & Define Business Area & TGSB \\
\hline & Define Functional Area & TFKB \\
\hline & Maintain consolidation business area & TGSBK \\
\hline & Maintain FM Area & FM01 \\
\hline \multirow{2}{*}{ Controlling } & Maintain Controlling Area & TKA01 \\
\hline & Create Operating Concern & TKEB \\
\hline \multirow{4}{*}{$\begin{array}{l}\text { Logistics - } \\
\text { General }\end{array}$} & Define valuation level & \\
\hline & Define, copy, delete, check plant & T001W \\
\hline & Define Location & T499S \\
\hline & Define, copy, delete, check division & TSPA \\
\hline \multirow{4}{*}{$\begin{array}{l}\text { Sales and } \\
\text { Distribution }\end{array}$} & Define, copy, delete, check sales organization & TVKO \\
\hline & Define, copy, delete, check distribution channel & TVTW \\
\hline & Maintain sales office & TVBUR \\
\hline & Maintain sales group & TVKGR \\
\hline \multirow{3}{*}{$\begin{array}{l}\text { Materials } \\
\text { Management }\end{array}$} & $\begin{array}{l}\text { Restrictions for the Industry Solution for Defense Forces \& Public } \\
\text { Security }\end{array}$ & \\
\hline & Maintain storage location & T001L \\
\hline & Maintain purchasing organization & T024E \\
\hline \multirow{5}{*}{$\begin{array}{l}\text { Logistics } \\
\text { Execution }\end{array}$} & Define, copy, delete, check warehouse number & T300 \\
\hline & $\begin{array}{l}\text { Restrictions for the Industry Solution for Defense Forces \& Public } \\
\text { Security }\end{array}$ & \\
\hline & Define, copy, delete, check shipping point & TVST \\
\hline & Maintain loading point & TVLA \\
\hline & Maintain transportation planning point & TTDS \\
\hline $\begin{array}{l}\text { Plant } \\
\text { Maintenance }\end{array}$ & Maintain maintenance planning plant & T3991 \\
\hline \multirow{4}{*}{$\begin{array}{l}\text { Human } \\
\text { Resources } \\
\text { Management }\end{array}$} & Personnel Areas & T500P \\
\hline & Personnel Subareas & T001P \\
\hline & Employee Groups & T501 \\
\hline & Employee Subgroups & T503K \\
\hline
\end{tabular}

\footnotetext{
${ }^{7}$ ABAP (Advanced Business Application Programming) is a programming language for developing applications in the SAP R/3 system. https://help.sap.com/saphelp_46c/helpdata/en/fc/eb2c46358411d1829f0000e829fbfe/frameset.htm

${ }^{8}$ SPRO - a four or more digit code used when working in SAP by which the user directly launches the given transaction (application) without having to click through what are often several levels of menus.
} 
Computer program for complexity calculation. In order to calculate complexity, the authors created the program IHC_ORG (Identify High Complexity), which counts the entries in the relevant tables (here the tables determine the settings for the enterprise structure) and at the same time aggregates them according to individual SAP modules. The result of the program is a single number which counts items in selected tables, or possibly set of numbers sorted by SAP modules to which the tables primarily belong. The program can be launched for one or multiple clients of the given system and then their complexity can be compared. On the program start screen (Figure 1) it is possible to pre-select the client (S_MANDT) or enter other tables as parameters (P_TABS). After run, the program displays all the tables and their entries in detail as shown on Figure 2. This can also serve to identify directly the settings which are causing a high complexity index.

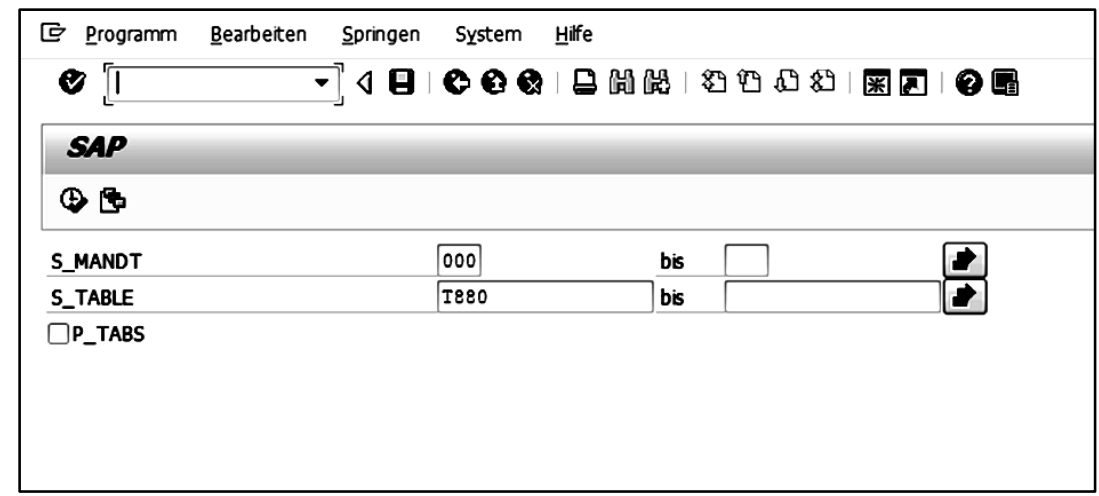

Figure 1. Start Screen of Identify High Complexity program

Data dimension. Another possible modification of the program is the variant for selecting essential system objects and subsequently calculating their instances. As an object we define a field in the database table that is found in multiple tables as a key field. For such a field we find all the control tables, i.e. tables where this field is the sole key. The number of entries of such tables is then multiplied by the number of instances of the given field (object) in the keys of the other tables. The resulting number is then divided by the number of tables in the database.

Using the program. The program can be installed into any SAP system. Because the program evaluates customisation tables, which are transported from the development system through the testing system to the productive system and are, thus, identical in all systems, the program can also be launched in the development system without having to transport it. The program can be downloaded from http://www.holub.cz.

Evaluation. The method, the program, and its usage were evaluated in a case study of division of one industrial company in Germany (Company A), which was divided to two companies (Companies B and C), of which one (C) was merged with another company (D). The result is described in a study [10]. The resulted measure was used to manage and decrease resulted organisation structure complexity of ERP implementation. The originally considered solution was to create a new client in the SAP System for the Company D. After calculation of the expected complexity the project manager decided to implement the structures of company D within the client of company $\mathrm{C}$ and to use most of common structures for both companies. The Figure 3 shows the result of measuring complexity of SAP systems of companies C and D. 


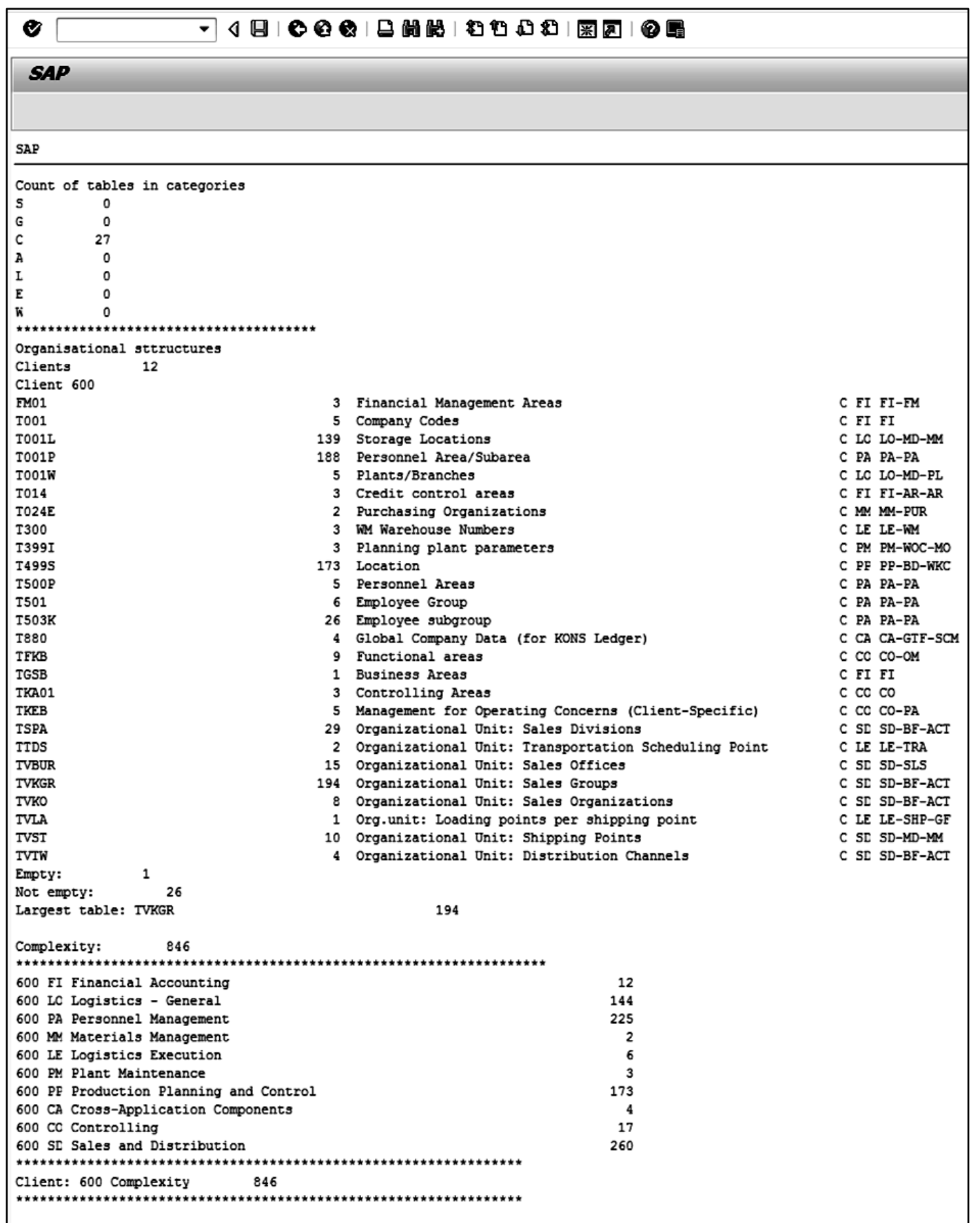

Figure 2. Output of the Identify High Complexity program

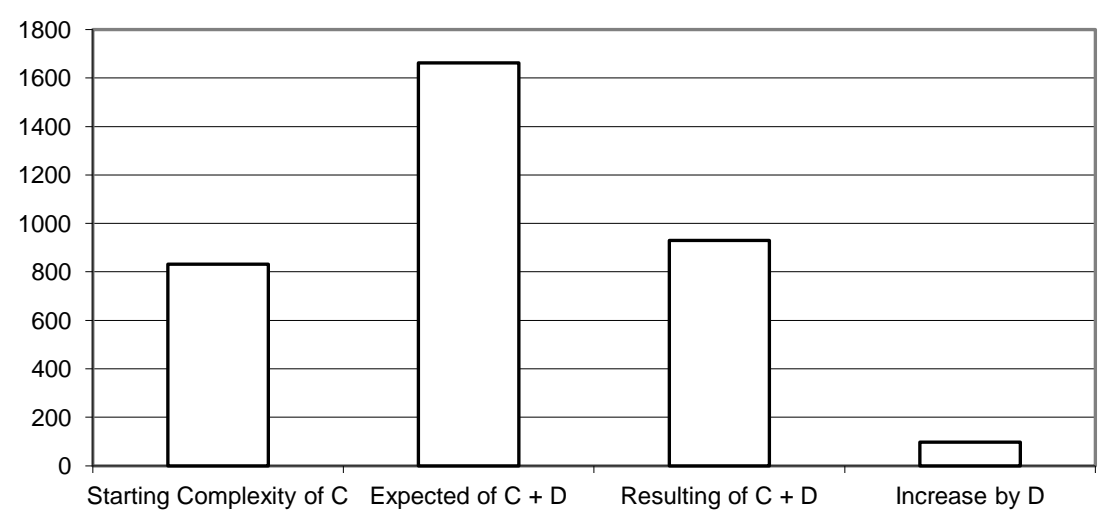

Figure 3. Organization structure complexity of the SAP system in Enterprises C and D 


\section{Conclusion}

We discussed the context of complexity in SAP systems, proposed a method for measuring complexity of running SAP R/3 ERP systems, and implemented the method in a software. The complexity of SAP systems is influenced by extension of SAP R/3 system, by other systems, and by duplication functionality and data structures outside the core ERP system. The simple measurement method can count selected items in the system from a chosen dimension, which provides a tool for observing complexity in time and during changes and for reducing complexity and keeping the system as simple as possible.

We validated the method and the program in SAP implementation project. The application of Identify High Complexity program did help to save costs and time of the ERP system implementation and made the maintenance of the systems easier.

While the method was described and exemplified and validated on SAP systems, it potentially could be useful also for managing complexity for other ERP Systems. The validation of this usefulness could be a subject of further research.

The extended abstract of this paper is available in [15].

\section{References}

[1] R. Diestel, "Graph Theory,” in Graduate Texts in Mathematics, 4th Edition. Springer, vol. 173, p. 410, 2010.

[2] M. Dehmer, "Structural Analysis of Complex Networks," in Structural Analysis of Complex Networks, 1st Edition. Birkhäuser, Boston, 2010. Available: http://dx.doi.org/10.1007/978-0-8176-4789-6

[3] V. Bansal and T. Negi, "A Metric for ERP Complexity," in Business Information Systems, proc. of the 11th International Conference, BIS 2008, Innsbruck, Austria, May 5-7, 2008. vol. 7, pp. 369-379, 2008. Availbale: http://dx.doi.org/10.1007/978-3-540-79396-0_32

[4] A. Phukan, M. Kalava and V. Prabhu, "Complexity Metrics for Manufacturing Control Architectures Based on Software and Information Flow," Comput. Ind. Eng., vol. 49, no. 1, pp. 1-20, 2005. Available: http://dx.doi.org/10.1016/j.cie.2005.01.005

[5] D. E. Geer, "Complexity Is the Enemy," Secur. Priv. IEEE, vol. 6, no. 6, p. 88, 2009. Available: http://dx.doi.org/10.1109/MSP.2008.139

[6] P. Mason and K. Cosh, "Managing Complexity in ICT Systems Development," Int. J. Inf. Technol. Manag., vol. 7, no. 3, pp. 264-282, 2008. Available: http://dx.doi.org/10.1504/IJITM.2008.017605

[7] J. Maeda, The Laws of Simplicity, MIT Press, p. 117, 2006.

[8] S. Henry, D. Hafura, "Software Structure Metrics Based on Information Flow," IEEE Trans. Softw. Eng., vol. SE-7, no. 5, IEEE Press Piscataway, NJ, USA, Sept. 1981.

[9] P. P. Lemberger and M. Morel, "Managing Complexity of Information Systems: The Value of Simplicity," 1st edition. Wiley-ISTE, p. 255, 2013.

[10] I. Holub, "Methodology for Measuring the Complexity of Enterprise Information Systems," J. Syst. Integr., vol. 7, no. 3, pp. 34-53, 2016. Available: http://dx.doi.org/10.20470/jsi.v7i3.260

[11] The World's Biggest Public Companies List - Forbes. [Online]. Available: http://www.forbes.com/global2000/list/\#industry:Software\%20\%26\%20Programming (accessed 07.05.2016)

[12] ERP System, Enterprise Resource Planning, SAP. [Online]. Available: http://go.sap.com/product/enterprisemanagement/erp.html (accessed 28.03.2016)

[13] DSAG, 13. DSAG-Jahreskongress in Bremen: Zurück zur alten Stärke Integration. DSAG, 25.09.2012. [Online]. Available: https://www.dsag.de/sites/default/files/PM_DSAG_JK_2012.pdf

[14] Cloud und Hana statt ERP: DSAG kritisiert SAP-Strategie, 2012. [Online]. Available: http://www.computerwoche.de/a/dsag-kritisiert-sap-strategie,2523716 (accessed 12.02.2013)

[15] I. Holub and T. Bruckner, "Measuring Complexity of SAP Systems," B. Johansson, F. Vencovský, Eds., Joint Proceedings of the BIR 2016 Workshops and Doctoral Consortium co-located with 15th International Conference on Perspectives in Business Informatics Research (BIR 2016), Prague, Czech Republic, September 14-16, 2016. [Online]. Available: http://ceur-ws.org/Vol-1684/paper15.pdf 